Appl. Math. Mech. -Engl. Ed., 42(8), 1183-1190 (2021)

APPLIED MATHEMATICS AND MECHANICS (ENGLISH EDITION)

https://doi.org/10.1007/s10483-021-2754-5

\title{
Horizontal convection in a rectangular enclosure driven by a linear temperature profile*
}

\author{
Tianyong YANG, Bofu WANG, Jianzhao WU`, Zhiming LU, Quan ZHOU \\ Shanghai Key Laboratory of Mechanics in Energy Engineering, \\ Shanghai Institute of Applied Mathematics and Mechanics, \\ School of Mechanics and Engineering Science, \\ Shanghai University, Shanghai 200072, China \\ (Received Mar. 29, 2021 / Revised Jun. 2, 2021)
}

\begin{abstract}
The horizontal convection in a square enclosure driven by a linear temperature profile along the bottom boundary is investigated numerically by using a finite difference method. The Prandtl number is fixed at 4.38, and the Rayleigh number $R a$ ranges from $10^{7}$ to $10^{11}$. The convective flow is steady at a relatively low Rayleigh number, and no thermal plume is observed, whereas it transits to be unsteady when the Rayleigh number increases beyond the critical value. The scaling law for the Nusselt number $N u$ changes from Rossby's scaling $N u \sim R a^{1 / 5}$ in a steady regime to $N u \sim R a^{1 / 4}$ in an unsteady regime, which agrees well with the theoretically predicted results. Accordingly, the Reynolds number $R e$ scaling varies from $R e \sim R a^{3 / 11}$ to $R e \sim R a^{2 / 5}$. The investigation on the mean flows shows that the thermal and kinetic boundary layer thickness and the mean temperature in the bulk zone decrease with the increasing $R a$. The intensity of fluctuating velocity increases with the increasing $R a$.
\end{abstract}

Key words horizontal convection, linear temperature profile, turbulent heat transfer, boundary layer

Chinese Library Classification $\mathrm{O} 357.5^{+} 3$

2010 Mathematics Subject Classification 76 F25

\section{Introduction}

There are three classical models for the study of thermal convection, i.e., Rayleigh-Bénard convection (RBC), vertical convection (VC), and horizontal convection (HC) ${ }^{[1]}$. In RBC systems, the bottom plate is heated, and the top plate is cooled, while in VC systems, the heating and cooling are respectively imposed on two parallel vertical side walls. $\mathrm{HC}$ is different from the former two, whose heating and cooling are in the same floor or roof. The study of HC is

* Citation: YANG, T. Y., WANG, B. F., WU, J. Z., LU, Z. M., and ZHOU, Q. Horizontal convection in a rectangular enclosure driven by a linear temperature profile. Applied Mathematics and Mechanics (English Edition), 42(8), 1183-1190 (2021) https://doi.org/10.1007/s10483-021-2754-5

$\dagger$ Corresponding author, E-mail: jianzhao_wu@shu.edu.cn

Project supported by the National Natural Science Foundation of China (Nos. 11988102, 92052201, 11972220, 11825204, 91852202, and 11732010), the China Postdoctoral Science Foundation (No. 2020M681259), and the Key Research Projects of Shanghai Science and Technology Commission (Nos. 19JC1412802 and 20ZR1419800)

(C) The Author(s) 2021 
of great significance in the fields of geophysical and geological flows, such as the study of planetary atmospheric flow and mixing ${ }^{[2]}$, the mass transport in river ${ }^{[3]}$, and the energy exchange of large-scale ocean circulation ${ }^{[4]}$, as well as in industrial applications like glass dissolution and manufacturing ${ }^{[5]}$.

A large number of studies have been focused on RBC and its derived configurations during past decades, for instance, $\mathrm{RBC}$ with rough boundaries ${ }^{[6-7]}$, control of $\mathrm{RBC}$ with sidewall temperature $^{[8]}, \mathrm{RBC}$ with external forcing ${ }^{[9-10]}$. However, $\mathrm{HC}$ has received much less attention. $\mathrm{HC}$ is often established in the laboratory via heating and cooling at the bottom boundary of an enclosure ${ }^{[11]}$. The fluid in $\mathrm{HC}$ is advected from a region of stable stratification to an unstable stratification where thermal plumes are vertically transported away from the boundary. The flow strength in $\mathrm{HC}$ is weaker than that in $\mathrm{RBC}$, which means that the convective flow becomes turbulent for a larger Rayleigh number $R a$ than in RBC. In another word, the critical $R a$ for the transition to turbulent $\mathrm{HC}$ is much higher than that for $\mathrm{RBC}^{[12]}$ or related thermal convections ${ }^{[13-14]}$. For instance, for $R a$ about $10^{9}$, the bulk flows in RBC are already turbulent ${ }^{[12,15-17]}$, while the HC flows are still laminar ${ }^{[18]}$.

Rossby ${ }^{[19]}$ made the first step to develop a model for the scaling behavior of the dimensionless Nusselt number $N u$ depending on $R a$, namely, $N u \sim R a^{1 / 5}$, where " $\sim$ " denotes the scaling relation. This is nowadays so called Rossby scaling. The derivation of this scaling is based on the assumption that the HC flows are laminar and the heat transport is mainly dominated by their boundary layers. This Rossby scaling is supported by several numerical simulations ${ }^{[18,20-22]}$ and laboratory experiments ${ }^{[21,23-25]}$. At a sufficiently large Rayleigh number, HC has been shown to become unsteady ${ }^{[18,26-27]}$. The scaling relation becomes $N u \sim R a^{1 / 4[28]}$. Sheard and King ${ }^{[29]}$ also reported an elevation of the Nusselt number scaling exponent beyond $R a^{1 / 5}$ for Rayleigh numbers $R a>10^{10}$ for HC heated by a linear thermal profile. At an extremely large Rayleigh number, Siggers et al. ${ }^{[26]}$ derived that the upper bound of the scaling exponent in $\mathrm{HC}$ which equals $1 / 3$ with variational analysis. Winters and Young ${ }^{[30]}$ estimated the upper bound of the mean thermal dissipation rate in $\mathrm{HC}$, which is also consistent with the theoretical result by Siggers et al. ${ }^{[26]}$. Shishkina et al. ${ }^{[31]}$ applied the ideas by Grossmann and Lohse ${ }^{[32]}$ for RBC to an $\mathrm{HC}$ configuration. They decomposed the global averaged kinetic and thermal dissipation rates into the contributions of the boundary layer and bulk zone. Various known scaling laws have been derived, i.e., the Rossby's scaling law in a laminar boundary layer dominated regime, the limiting scaling proposed by Siggers et al. ${ }^{[26]}$ referred to as the ultimate regime, and several regimes that predict a Nusselt number scaling going with $R a^{1 / 4}$. Moreover, various new limiting scaling laws have also been revealed.

Note that although there are different driving temperature variations imposed throughout the literature, HC nevertheless tends to exhibit consistent behavior with increased Rayleigh numbers. The aim of this study is to investigate the flow structure and heat transport of $\mathrm{HC}$ in a square cavity imposed with a linear temperature profile. It is expected to shed more light on the robustness of Nusselt numbers and Reynolds number scalings at various Rayleigh numbers as well as under this specific temperature condition.

This paper is structured as follows. Section 2 gives a detailed description of the physical model and numerical method. This is followed in Section 3 by results of flow structures, global features, and mean flow properties, while conclusions are drawn in Section 4.

\section{Physical model and numerical method}

In this study, we investigate the flow structures and heat transport in HC within a square enclosure of size $H$. The bottom of the enclosure is imposed with a linear temperature profile with temperature difference of $\Delta$, and the top as well as the two sidewalls is insulated as shown in Fig. 1. It is worth noting that different from RBC and VC where both the heating and cooling promote overturning of the fluid in the box, in the present model, only linear heating promotes such overturning. Such a difference in HC leads to the intriguing flow dynamics. 
The dimensionless Oberbeck-Boussinesq equations for $\mathrm{HC}$ read

$$
\begin{aligned}
& \nabla \cdot \boldsymbol{u}=0, \\
& \partial_{t} \boldsymbol{u}+(\boldsymbol{u} \cdot \nabla) \boldsymbol{u}=-\nabla p+\sqrt{\frac{P r}{R a}} \nabla^{2} \boldsymbol{u}+\theta \boldsymbol{e}_{z}, \\
& \partial_{t} \theta+(\boldsymbol{u} \cdot \nabla) \theta=\frac{1}{\sqrt{\operatorname{RaPr}}} \nabla^{2} \theta,
\end{aligned}
$$

where $\boldsymbol{u}(\boldsymbol{x}, t)$ denotes the fluid velocity non-dimensionalized by the free fall velocity $\sqrt{\alpha g \Delta H}$, $t$ is the dimensionless time normalized by $\sqrt{H /(\alpha g \Delta)}, \theta(\boldsymbol{x}, t)$ denotes the temperature nondimensionalized by the temperature difference $\Delta$ at the bottom plate, and $p(\boldsymbol{x}, t)$ denotes the dimensionless kinematic pressure field. Here, $\alpha$ is the volumetric coefficient of thermal expansion, and $g$ is the gravitation magnitude. As shown in Fig. $1, \boldsymbol{x}=(x, z)$ represents the coordinate system, and $\boldsymbol{e}_{x}$ and $\boldsymbol{e}_{z}$ are the corresponding unit vectors. The velocity components are expressed by $u$ in the $x$-direction and by $v$ in the $z$-direction, i.e., $\boldsymbol{u}=(u, v)$. For the HC system, the relevant control parameters are the Rayleigh number $R a$ (measuring the strength of thermal driving) and the Prandtl number Pr. They are expressed by

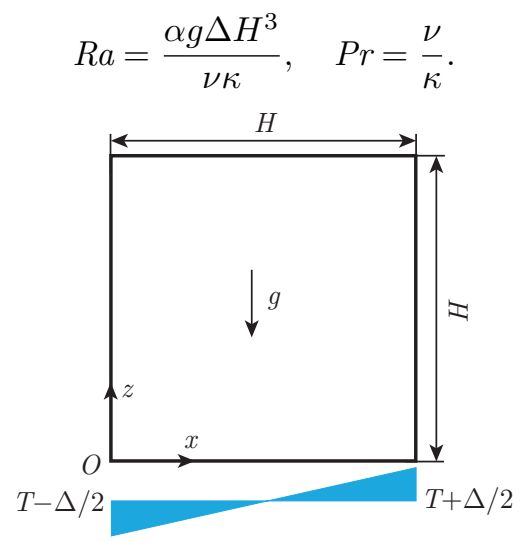

Fig. 1 Sketch of HC in a square enclosure, i.e., the flow is driven by a linear temperature profile with temperature difference of $\delta T$ from below, and the other three walls are insulated

Similar to the RBC system, there are two key response parameters in HC, i.e., the Nusselt number measuring the dimensionless heat transport flux and the Reynolds number characterizing the extent of flow. These two numbers are respectively defined by

$$
N u=-\left\langle\frac{\partial \theta}{\partial z}\right\rangle_{+}=\left\langle\frac{\partial \theta}{\partial z}\right\rangle_{-}, \quad R e=\sqrt{R a / \operatorname{Pr}} U_{\mathrm{rms}},
$$

where $\langle\cdot\rangle_{+}$and $\langle\cdot\rangle_{-}$mean the horizontally- and temporally-averagings of right half (hot) part and left half (cold) part of the bottom plate, respectively, and $U_{\mathrm{rms}}=\sqrt{\left\langle u^{2}+v^{2}\right\rangle}$ measures the magnitude of convective flows with $\langle\cdot\rangle$ denoting the globally- and temporally-averagings.

We solve the coupled equations of the velocity and temperature using a conservative finitedifference code, which has been detailedly described and well validated in Ref. [33]. The spatial derivative terms are approximated by a second-order central difference scheme with nonuniform staggered $\operatorname{grids}^{[34]}$. A fractional step approach is used to solve the momentum and temperature equations, and a multi-grid strategy is implemented to accelerate the iteration process in the Poisson equation. No-slip conditions are used for the velocity at all the walls. The temperature boundary conditions $\theta=x-0.5$ on the bottom and $\frac{\partial \theta}{\partial n}=0$ on the other walls are adopted. The number of grid points increases from $192 \times 192$ at $R a=10^{7}$ to $1536 \times 1536$ at $R a=10^{11}$ and is fixed at $192 \times 192$ for $R a<10^{7}$, to achieve a full resolution of HC. All statistics in this work are calculated over an averaging time of more than 600 free fall times units after the run has reached the statistically steady state. 


\section{Results}

\subsection{Flow structure}

Figure 2 depicts the instantaneous temperature contour overlaid with the velocity streamlines for $\mathrm{HC}$ at three different Rayleigh numbers, i.e., $R a=10^{4}, 10^{8}$, and $10^{11}$. It is seen in Fig. 2(a) that HC is diffusion-dominated at a low Rayleigh number $R a=10^{4}$. The heat transfer into the enclosure is dominated by molecular conduction from the wall to the adjacent fluid. With an increase in the Rayleigh number, the strength of thermal forcing increases and results in a horizontal thermal boundary layer, which transports the heat along the bottom wall leading to a convection-dominated flow within the enclosure. The convection is steady at a moderate Rayleigh number $R a=10^{8}$ as shown in Fig. 2(b). It can be observed that there exists a thermal boundary layer along the bottom plate, transporting the cold fluids (left) toward the hot zones (right). We can also see that the streamlines are clustered toward the hot side of the enclosure to form a vertical thermal pathway to transport the hot fluid toward the top boundary. The hot fluid then moves horizontally along the top boundary toward the colder side of the enclosure. This cooling effect eventually brings the fluid down over the cold stable boundary layer along the lower boundary. Thus, a convection roll is formed. With a further increase in Rayleigh number, the flow becomes unsteady, and an example of the flow structure at $R a=10^{11}$ is shown in Fig. 2(c). At this high Rayleigh number, the convective forcing is strong enough to trigger plume-like structures near the hot wall. However, these thin plumes do not have sufficient energy to reach the top boundary directly. Instead, they are convected horizontally to the hot end and rise along the side wall. It can also be observed that there are several corner rolls developed in this unsteady convection-dominated flow.

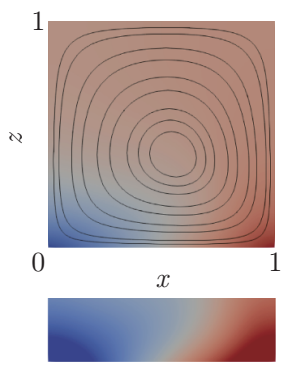

(a)

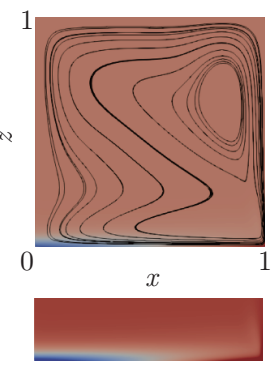

(b)

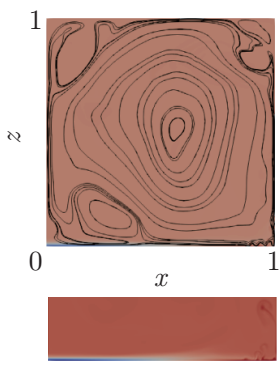

(c)

Fig. 2 Contour plots of temperature overlaid with velocity streamlines for horizontal convection at Rayleigh numbers $R a=$ (a) $10^{4}$, (b) $10^{8}$, and (c) $10^{11}$. Red and blue contours show arbitrary levels of warm and cool fluids, respectively. A close-up plot of the temperature distribution near bottom $(0 \leqslant z \leqslant 0.28,0 \leqslant x \leqslant 1)$ is drawn below with the temperature contour ranging from -0.3 to 0.3 (color online)

\section{$3.2 N u \sim R a$ and $R e \sim R a$ scalings}

The scaling behaviors of the Nusselt number $N u(R a)$ for the heat transport mechanism and the Reynolds number $R e(R a)$ associated with the large-scale circulation (LSC) are concerned in HC. The mean Nusselt number is calculated in the present configuration by computing the average temperature gradient normal to the wall along either the cold half part or the hot half one of the bottom, as defined in Eq. (5).

The calculated Nusselt numbers are plotted against the Rayleigh number in Fig. 3(a). Note that the Nusselt number is independent of the Rayleigh number in a diffusion-dominated regime and is not shown in the figure. The plot shows the variation of $N u$ for Rayleigh number from $10^{7}$ to $10^{11}$. It can be observed that the Nusselt numbers appear to scale with the Rayleigh number to the one-fifth power, i.e., $N u \sim R a^{1 / 5}$, for $10^{7} \leqslant R a \leqslant 1.4 \times 10^{9}$. This scaling is in agreement with the theoretical prediction by Rossby ${ }^{[19]}$ and subsequent experimental ${ }^{[8]}$ and numerical ${ }^{[14,21]}$ results. The scaling exponent slightly increases to $1 / 4$ for $1.4 \times 10^{9} \leqslant R a \leqslant 10^{11}$. Note that the 
flow is steady for $R a \leqslant 1.4 \times 10^{9}$, and it becomes unsteady at a higher $R a$. The variations of the scaling relationships are associated with this flow transition. The $N u \sim R a^{1 / 4}$ has been reported by Sheard and King ${ }^{[29]}$, and the variation is attributed to the development of unsteady flow in the enclosure. Moreover, the scaling relations between $R e$ and $R a$ are also different in these two regimes as illustrated in Fig. 3(b). The Reynolds numbers approximately scale with the Rayleigh number to the three-eleventh power, i.e., $R e \sim R a^{3 / 11}$ in the steady regime, and the scaling becomes $R e \sim R a^{2 / 5}$ in the unsteady regime. This indicates that both the flow structure characteristics and the heat transport mechanism are essentially different between the steady and unsteady regimes. Moreover, the compensated plots shown in Figs. 3(c) and 3(d) confirm the scaling behaviors in Figs. 3(a) and 3(b), respectively.

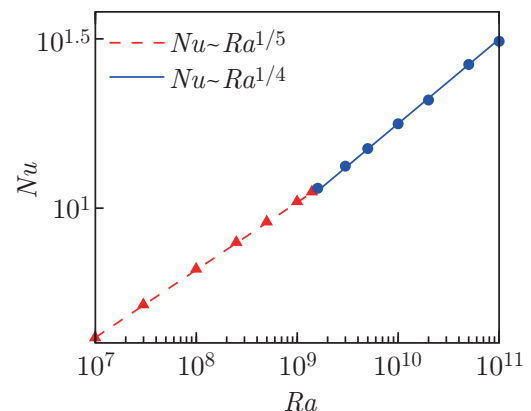

(a)

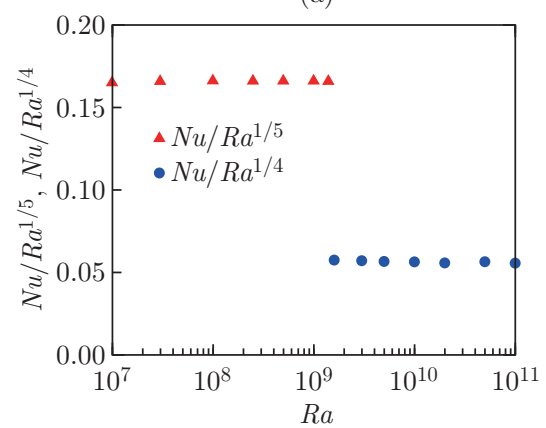

(c)

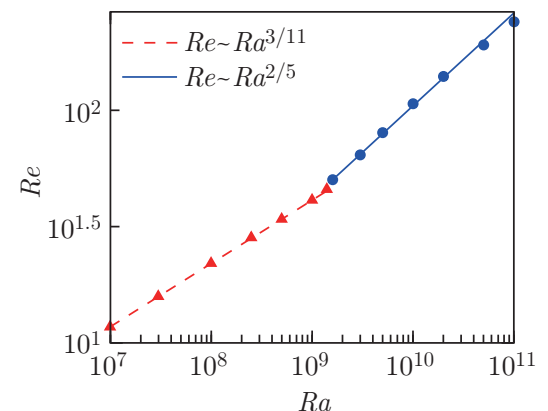

(b)

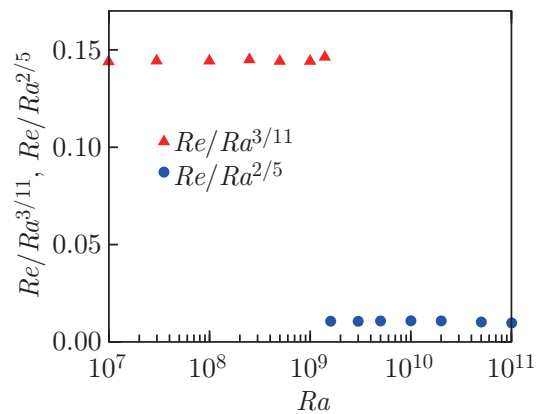

(d)

Fig. 3 Scaling behaviors in HC. (a) $N u \sim R a$ scaling and (b) $R e \sim R a$ scaling. The red dashed lines are the power-law fits $N u \sim R a^{1 / 5}$ and $R e \sim R a^{3 / 11}$ for the steady regime; the solid blue lines are the power-law fits $N u \sim R a^{1 / 4}$ and $R e \sim R a^{2 / 5}$ for the unsteady regime. The compensated plots of the scaling relationships in (a) and (b) are drawn in (c) and (d), respectively (color online)

\subsection{Mean flow properties}

Two mean temperature profiles, i.e., $\theta_{\text {cold }}(z)$ and $\theta_{\text {hot }}(z)$ can be obtained by averaging the time-averaged temperature field over the cold half and the hot half of the bottom. The profiles for various $R a$ are shown in Fig. 4. It can be seen from the $\theta_{\text {cold }}(z)$ shown in Fig. 4(a) that the mean temperature increases with $z$ and reaches a nearly constant value in the bulk for all the $R a$. In contrast, the $\theta_{\text {hot }}(z)$ shown in Fig. 4(b) decreases with increasing $z$ to a minimum and then increases to a near-constant bulk temperature. The bulk temperature in both $\theta_{\text {cold }}(z)$ and $\theta_{\text {hot }}(z)$ decreases with increasing $R a$. It can also be observed from $\theta_{\text {cold }}(z)$ and $\theta_{\text {hot }}(z)$ that the thermal boundary layer thickness deceases with an increasing $R a$.

In order to investigate the temperature and velocity distribution in the bulk, we plot in Fig. 5 the time-averaged horizontal profiles of temperature and vertical velocity obtained at the mid-height of the convection cell at various $R a$. The mean temperature at the mid-height decreases with the increasing $R a$, as shown in Fig. 5(a), which is in agreement with the trend of temperature profiles shown in Fig. 4. For all $R a$ studied, the vertical velocity is positive near 


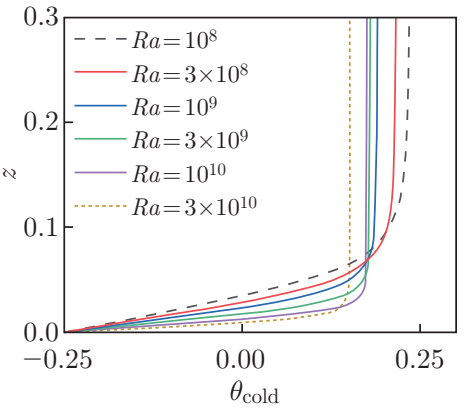

(a)

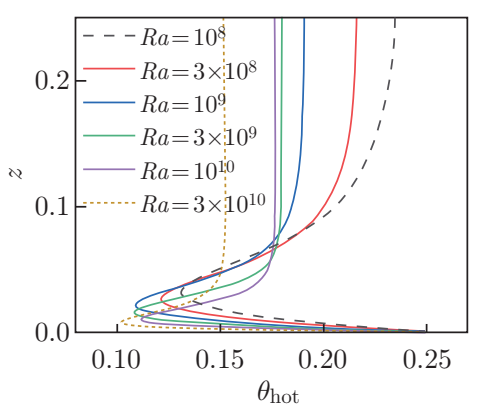

(b)

Fig. 4 Mean temperature profiles averaged over (a) the cold half and (b) the hot half of the bottom (color online)

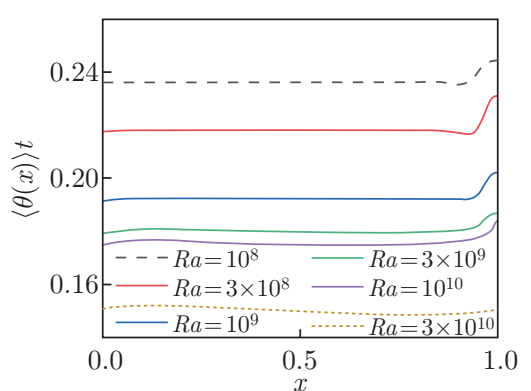

(a)

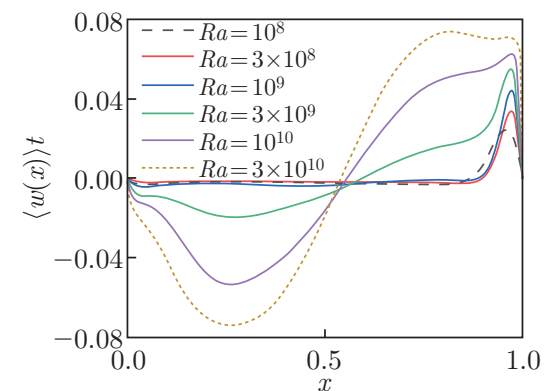

(b)

Fig. 5 The time-averaged horizontal profiles of (a) temperature and (b) vertical velocity obtained at the mid-height of the enclosure (color online)

the right wall, and negative near the left wall, as shown in Fig. 5(b), which is associated with the upwelling of hot fluids at the right side and downwelling of cold fluids at the left side under the driving of the LSC (see Fig. 2). Meanwhile, the magnitude of vertical velocity increases with $R a$. This indicates that with the increasing $R a$, the LSC moves much faster and contains the fluid that is much hotter or colder than the surrounding background, consequently leading to the enhanced mixing and lower bulk temperature.

We also compare vertical root-mean-square (RMS) profiles of horizontal velocity for different $R a$ in Fig. 6. The velocity RMS values are defined as follows:

$$
u_{\mathrm{RMS}}(z)=\sqrt{\left\langle u^{2}\right\rangle_{A, t}}
$$

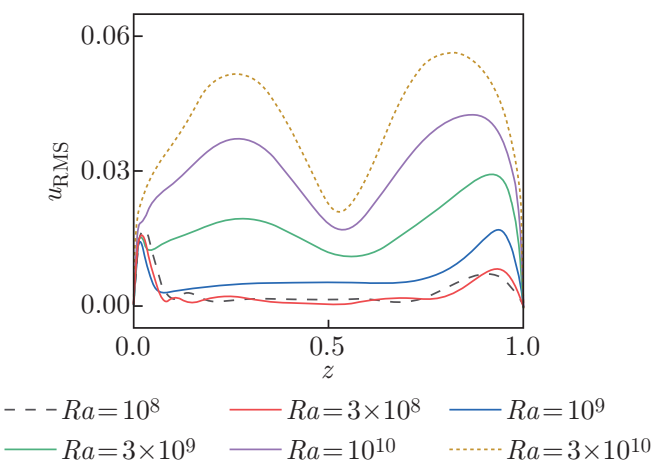

Fig. 6 RMS profiles of horizontal velocity in horizontal convection (color online) 
where $\langle\cdot\rangle_{A, t}$ stands for a combined average over time and horizontal planes. It is shown that the horizontal velocity fluctuation is stronger for the near-wall regions than that for the bulk for low $R a$ cases, while for high $R a$ cases, the horizontal velocity fluctuations are stronger for the region between the near-wall region and the bulk as revealed from Fig. 6. It should be noted that the temperature fluctuation is too weak to be presented.

\section{Conclusions}

In summary, the flow structures and heat transport of $\mathrm{HC}$ in a square enclosure are numerically studied for $\operatorname{Pr}=4.38$ and $10^{7} \leqslant R a \leqslant 10^{11}$. It is found that the convective flow is steady for $R a$ below $1.4 \times 10^{9}$ and unsteady for higher $R a$. Under the driving of linear temperature profile on the bottom plate, a vertical thermal pathway is formed to transport the hot fluids on the right side of unstable temperature gradient in the steady regime; with the increasing $R a$, the flow becomes unsteady, the convective forcing is strong enough to trigger thermal plumes for the thermal boundary layer on the unstable side, which strengthens the heat transport mechanism. The different flow features in these two regimes result in different $N u \sim R a$ and $R e \sim R a$ scaling relations, i.e., it is obtained $N u \sim R a^{1 / 5}$ and $R e \sim R a^{3 / 11}$ in the steady regime; whereas $N u \sim R a^{1 / 4}$ and $R e \sim R a^{2 / 5}$ in the unsteady regime. It is shown that with the increasing $R a$, both temperature and velocity boundary thickness decrease. It is also shown that the mean velocity in the bulk zone increases with the increasing $R a$, which leads to enhanced mixing with lower averaged temperature in the bulk region. In addition, the intensity of fluctuating velocity within $\mathrm{BL}$ and in the bulk increases with the increasing $R a$, but it is found that the fluctuating temperature is very weak even in $\mathrm{HC}$ at $R a=10^{11}$.

\section{References}

[1] HUGHES, G. O. and GRIGGiths, R. W. Horizontal convection. Annual Review of Fluid Mechanics, 40, 185-208 (2008)

[2] HOUGHTON, J. The Physics of Atmospheres, Cambridge University Press, London (2002)

[3] WANG, K. P., LI, Q. X., and DONG, Y. H. Transport of dissolved oxygen at the sediment-water interface in the spanwise oscillating flow. Applied Mathematics and Mechanics (English Edition), 42(4), 527-540 (2021) https://doi.org/10.1007/s10483-021-2719-6

[4] CUSHMANROISIN, B. and BECKERS, J. M. Introduction to Geophysical Fluid Dynamics, Academic Press, Salt Lake City (2011)

[5] COOPER, A. R. JR and KINGERY, W. D. Dissolution in ceramic systems: I, molecular diffusion, natural convection, and forced convection studies of sapphire dissolution in calcium aluminum silicate. Journal of the American Ceramic Society, 47(1), 37-43 (1964)

[6] ZHANG, Y. Z., SUN, C., BAO, Y., and ZHOU, Q. How surface roughness reduces heat transport for small roughness heights in turbulent Rayleigh-Bénard convection. Journal of Fluid Mechanics, 836, R2 (2018)

[7] DONG, D. L., WANG, B. F., DONG, Y. H., HUANG, Y. X., and ZHOU, Q. Influence of spatial arrangements of roughness elements on turbulent Rayleigh-Bénard convection. Physics of Fluids, 32, $045114(2020)$

[8] ZHANG, S., CHEN, X., XIA, Z., XI, H. D., and CHEN, S. Stabilizing/destabilizing the large-scale circulation in turbulent Rayleigh-Bénard convection with sidewall temperature control. Journal of Fluid Mechanics, 915, A14 (2021)

[9] WANG, B. F., ZHOU, Q., and SUN, C. Vibration-induced boundary-layer destabilization achieves massive heat-transport enhancement. Science Advances, 6, eaaz8239 (2020)

[10] WU, J. Z., DONG, Y. H., WANG, B. F., and ZHOU, Q. Phase decomposition analysis on oscillatory Rayleigh-Bénard turbulence. Physics of Fluids, 33, 045108 (2021)

[11] TSAI, T., HUSAM, W. K., KING, M. P., and SHEARD, G. J. Transitions and scaling in horizontal convection driven by different temperature profiles. International Journal of Thermal Sciences, 148, 106166 (2020) 
[12] WAGNER, S. and SHISHKINA, O. Aspect ratio dependency of Rayleigh-Bénard convection in box-shaped containers. Physics of Fluids, 25, 085110 (2013)

[13] XIONG, X. M. and TAO, J. J. Lower bound for transient growth of inclined buoyancy layer. Applied Mathematics and Mechanics (English Edition), 38(6), 779-796 (2017) https://doi.org/10.1007/s10483-017-2202-8

[14] SUN, C. S., LIU, S., WANG, Q., WAN, Z. H., and SUN, D. J. Bifurcations in penetrative Rayleigh-Bénard convection in a cylindrical container. Applied Mathematics and Mechanics (English Edition), 40(5), 695-704 (2019) https://doi.org/10.1007/s10483-019-2474-6

[15] XU, A., SHI, L., and XI, H. D. Lattice Boltzmann simulations of three-dimensional thermal convective flows at high Rayleigh number. International Journal of Heat and Mass Transfer, 140, 359-370 (2019)

[16] LIU, S. and HUISMAN, S. Heat transfer enhancement in Rayleigh-Bénard convection using a single passive barrier. Physical Review Fluids, 5(12), 123502 (2020)

[17] YANG, J. L., ZHANG, Y. Z., JIN, T. C., DONG, Y. H., WANG, B. F., and ZHOU, Q. The Pr-dependence of the critical roughness height in two-dimensional turbulent Rayleigh-Bénard convection. Journal of Fluid Mechanics, 911, A52 (2021)

[18] GAYEN, B., GRIFFITHS, R., and HUGHES,G. Stability transitions and turbulence in horizontal convection. Journal of Fluid Mechanics, 751, 698-724 (2014)

[19] ROSSBY, H. T. On thermal convection driven by non-uniform heating from below: an experimental study. Deep-Sea Research and Oceanographic Abstracts, 12(1), 9-16 (1965)

[20] ROSSBY, H. T. Numerical experiments with fluid heated non-uniformly from below. Tellus A, 50, 242-257 (1998)

[21] MULLARNEY, J., GRIGGITHES, R., and HUGHES, G. Convection driven by differential heating at a horizontal boundary. Journal of Fluid Mechanics, 516, 181-209 (2004)

[22] CHIU-WEBSTER, S., HINCH, J., and LISTER, J. Horizontal infinite-Prandtl-number convection. 59th Annual Meeting of the APS Division of Fluid Dynamics, American Physical Society, Tampa Bay, Florida (2006)

[23] WANG, W. and HUANG, R. X. An experimental study on thermal circulation driven by horizontal differential heating. Journal of Fluid Mechanics, 540, 49-73 (2005)

[24] HUGhes, G. O., GRIfFiths, R. W., MUllarney, J. C., and PETERSON, W. H. A theoretical model for horizontal convection at high Rayleigh number. Journal of Fluid Mechanics, 581, 251-276 (2007)

[25] STEWART, K. D., HUGHES, G. O., and GRIFFITHS, R. W. The role of turbulent mixing in an overturning circulation maintained by surface buoyancy forcing. Journal of Physical Oceanography, 42(11), 1907-1922 (2012)

[26] SIGGERS, J. H., KERSWELL, R. R., and BALMFORTH, N. J. Bounds on horizontal convection. Journal of Fluid Mechanics, 517, 55-70 (2004)

[27] COMAN, M. A., GRIFFITHS, R. W., and HUGHES, G. O. The sensitivity of convection from a horizontal boundary to the distribution of heating. Journal of Fluid Mechanics, 647, 71-90 (2010)

[28] SHISHKINA, O. and WAGNER, S. Prandtl-number dependence of heat transport in laminar horizontal convection. Physical Review Letters, 116(2), 024302 (2016)

[29] SHEARD, G. J. and KING, M. P. Horizontal convection: effect of aspect ratio on Rayleigh number scaling and stability. Applied Mathematical Modelling, 35(4), 1647-1655 (2011)

[30] WINTERS, K. B. and YOUNG, W. R. Available potential energy and buoyancy variance in horizontal convection. Journal of Fluid Mechanics, 629, 221-230 (2009)

[31] SHISHKINA, O., GROSSMANN, S., and LOHSE, D. Heat and momentum transport scalings in horizontal convection. Geophysical Research Letters, 43(3), 1219-1225 (2016)

[32] GROSSMANN, S. and LOHSE, D. Scaling in thermal convection: a unifying view. Journal of Fluid Mechanics, 407, 27-56 (2000)

[33] WANG, Q., XU, B. L., XIA, S. N., WAN, Z. H., and SUN, D. J. Thermal convection in a tilted rectangular cell with aspect ratio 0.5. Chinese Physics Letters, 34(10), 104401 (2017)

[34] ZHANG, Y. Z., XIA, S. N., DONG, Y. H., WANG, B. F., and ZHOU, Q. An efficient parallel algorithm for DNS of buoyancy-driven turbulent flows. Journal of Hydrodynamics, 31, 1159-1169 (2019) 